\title{
Impacto da atividade física e esportes sobre o crescimento e puberdade de crianças e adolescentes
}

\author{
Linear growth and puberty in children and adolescents: effects of physical activity and sports
}

\section{Crésio Alves ${ }^{1}$, Renata Villas Boas Lima²}

\section{RESUMO}

Objetivo: Apresentar revisão atualizada e crítica sobre o impacto do esporte e da atividade física no crescimento, desenvolvimento puberal e mineralização óssea de crianças e adolescentes.

Fontes de dados: Pesquisa bibliográfica nos bancos de dados Medline e Lilacs (1987-2007), selecionando os artigos escritos em inglês, português ou espanhol, a partir dos descritores "esportes" e "exercícios", em combinação com "crescimento", "puberdade" e "mineralização óssea". Foram examinados 252 artigos e 48 deles selecionados.

Síntese dos dados: Diferentes modalidades esportivas não aumentam ou diminuem a estatura. Ocorre um viés de seleção, no qual fatores constitucionais determinam a seleção de biótipos privilegiados para determinados esportes. O exercício físico leve a moderado estimula o crescimento e deve ser incentivado. A atividade física extenuante, principalmente quando associada à restrição dietética, afeta o crescimento, o desenvolvimento puberal, a função reprodutiva e a mineralização óssea. A musculação praticada por jovens pré-púberes pode ser prejudicial, se não for realizada sob supervisão, já que há um potencial risco de lesão na cartilagem de crescimento. Entretanto, quando bem supervisionada, pode levar a um aumento de força e resistência muscular.

Conclusões: Os efeitos deletérios dos esportes sobre o crescimento e desenvolvimento só foram observados em atletas de elite submetidos a treinamento intensivo e restrição alimentar. Alterações hormonais e de citocinas inflamatórias são parte da fisiopatologia desse processo. É necessário que estudos longitudinais avaliem as repercussões da atividade física recreacional sobre a estatura final.
Palavras-chave: esportes; exercício; crescimento; puberdade; criança; adolescente.

\section{ABSTRACT}

Objective: To present an up-to-date critical review about the impact of sports and physical activity on growth, pubertal development and bone mineralization of children and adolescents.

Data source: Bibliographic search of Medline and Lilacs databases (1987-2007) with selection of studies written in English, Portuguese or Spanish, with the descriptors "sports" and "exercise" in combination with "growth", "puberty" and "bone mineralization". A total of 252 articles were retrieved and 48 of them were selected.

Data synthesis: Light to moderate physical activity has a beneficial effect on growth and bone development, while intense physical training, specially if associated to dietary restrictions, may attenuate linear growth, pubertal development, reproductive function and bone mineralization. Different sports do not have specific effects on final height. There is a selection bias in which constitutional factors contribute to select favored biotypes to specific sports. Weight-training by pre-pubertal children may be harmful, if not supervised, due to the potential risk of injure to the growth plates; however, if performed under strict supervision, may provide muscle strength and resistance.

Conclusions: Deleterious effects of sports in growth and development of children and adolescents were only observed in elite athletes submitted to intensive training and dietetic restriction. Longitudinal studies are neded to
Instituição: Serviço de Endocrinologia Pediátrica do Hospital Universitário Professor Edgard Santos (Upes) da Faculdade de Medicina da Universidade Federal da Bahia (UFBA), Salvador, BA, Brasil

'Doutor, professor de Pediatria, coordenador da Residência Médica em Endocrinologia Pediátrica do Hospital Upes da Faculdade de Medicina da UFBA, Salvador, Bahia, Brasil

${ }^{2}$ Médica residente em Endocrinologia Pediátrica no Hospital Upes da Faculdade de Medicina da UFBA, Salvador, Bahia, Brasil
Endereço para correspondência:

Doutor Crésio Alves

Rua Plínio Moscoso, 222, apto. 601

CEP 40157-190 - Salvador/BA

E-mail: cresio.alves@uol.com

Recebido em: 14/4/2008

Aprovado em: 18/6/2008 
evaluate the effects of recreational physical activity upon linear growth.

Key-words: sports; exercise; growth; puberty; child; adolescents.

\section{Introdução}

Vários estudos com crianças e adolescentes têm demonstrado o benefício da atividade física no estímulo ao crescimento e desenvolvimento, prevenção da obesidade, incremento da massa óssea, aumento da sensibilidade à insulina, melhora do perfil lipídico, diminuição da pressão arterial, desenvolvimento da socialização e da capacidade de trabalhar em equipe $^{(1,2)}$. Também é conhecido o fato de que a atividade física realizada de forma imprópria, em desacordo com a idade, com o desenvolvimento motor e com o estado de saúde, apresenta riscos de lesões como: trauma, osteocondrose, fratura e disfunção menstrual ${ }^{(1,3)}$.

O que não está claro e se tornou motivo de conflito entre pais, pacientes e médicos é a definação de qual seria o melhor esporte ou atividade física para estimular o crescimento e o desenvolvimento de crianças e adolescentes. Nos consultórios são comuns questões como: "Que esporte o senhor(a) recomenda para ajudar meu filho com baixa estatura a crescer mais?"; "Meu filho já pode fazer musculação?”; "É verdade que ginástica olímpica diminui e o basquete aumenta a previsão de altura final?"; "Dançarinas de balé deixam de menstruar?"; "A atividade física melhora o desenvolvimento ósseo?”.

Esse estudo tem o objetivo de fazer uma revisão crítica e atualizada sobre o impacto do esporte (prática metódica de exercícios, individual ou coletivamente, com finalidade competitiva) e atividade física (prática de exercícios, individual ou coletivamente, sem finalidade competitiva) no crescimento, desenvolvimento puberal e mineralização óssea de crianças e adolescentes.

\section{Métodos}

Artigos científicos publicados nos últimos 20 anos abordando a associação de esportes e atividade física ao crescimento e ao desenvolvimento de crianças e adolescentes foram pesquisados através dos bancos de dados Medline/PubMed e Lilacs/Bireme. Na pesquisa bibliográfica foram utilizados os seguintes unitermos em várias combinações: "sports", "exercice", "athlete", "child", "adolescent" e "bone mineralization".
A pesquisa bibliográfica incluiu consensos, editoriais, estudos de coorte, estudos transversais, de revisão, casoscontrole e ensaios clínicos escritos nas línguas inglesa, portuguesa ou espanhola. Os artigos foram inicialmente selecionados por meio de seus títulos e resumos. O desfecho desejado era o impacto de atividades físicas e esportes sobre o crescimento, desenvolvimento e mineralização óssea de crianças e adolescentes. Após a leitura do resumo, os artigos selecionados foram obtidos através do Portal de Periódico da Capes ou nas Bibliotecas da Faculdade de Medicina da Bahia da Universidade Federal da Bahia (UFBA) e do Hospital São Rafael. Os artigos não obtidos por essas fontes foram solicitados à Bireme. Foram excluídos da revisão estudos não relacionados à faixa etária pediátrica ou a adolescentes, não escritos nos idiomas selecionados, artigos sem resumo ou cujo título não estivesse relacionado aos objetivos da revisão. De um total de 252 artigos, 48 preencheram os critérios de inclusão.

\section{Atividade física, esportes e desenvolvimento ósseo}

A densidade mineral óssea atinge cerca de $90 \%$ do seu pico no final da segunda década. Um quarto do osso adulto é acumulado durante os dois anos de pico de velocidade de crescimento $^{(4)}$. Fatores endógenos e exógenos participam desse processo.

Dentre os fatores endógenos destacam-se: genética, raça, aumento dos hormônios anabólicos associados à puberdade (hormônio do crescimento $[\mathrm{GH}]$, fator de crescimento insuliniforme tipo 1 [IGF-1], esteróides sexuais) e marcadores de remodelação óssea ${ }^{(3-5)}$. Receptores de leptina têm sido encontrados no tecido ósseo, sugerindo que este hormônio esteja envolvido na regulação esquelética ${ }^{(6)}$. No estudo de Muñoz et al, níveis séricos de leptina diminuiram significantemente em ginastas e bailarinas em comparação ao grupo controle ${ }^{(7)}$.

Em relação aos fatores exógenos (ambientais), destacam-se o exercício e a nutrição, com adequado aporte de cálcio na $\operatorname{dieta}^{(5,7,8)}$. Durante a atividade física, a contração muscular promove um aumento da atividade osteoblástica na região óssea próxima aos locais onde os músculos se inserem, levando ao aumento da mineralização óssea ${ }^{(9)}$. Por outro lado, a ausência de contração muscular, como nas situações de imobilização (por exemplo, paraplegia, fraturas) e de força gravitacional (por exemplo, vôos espaciais), causa significativa perda óssea ${ }^{(4)}$.

Os mecanismos de carga impostos pelos exercícios aumentam a densidade mineral óssea independentemente do sexo e da 
idade de quem os pratica ${ }^{(9)}$. Porém, o exercício físico realizado próximo ao pico máximo da velocidade de crescimento, ou seja, no início da puberdade, é mais efetivo para potencializar o ganho de massa óssea ${ }^{(1,9)}$. Os efeitos osteogênicos dos exercícios dependem ainda da magnitude da carga e da freqüência de aplicação que, quando repetidas, resultam em hipertrofia óssea ${ }^{(9)}$. Dessa forma, atividade física regular durante a infância e adolescência pode atuar na prevenção de distúrbios ósseos, como a osteoporose. O treinamento de força com impacto (por exemplo, corrida, ginástica, dança, basquetebol, atletismo) proporciona maior incremento da densidade mineral óssea comparado ao de resistência aeróbica, como ocorre na natação e no pólo aquático. Ambos os tipos de treinamento promovem aumento da densidade mineral óssea em comparação à nãorealização de qualquer uma dessas atividades ${ }^{(5,9)}$.

Enquanto a atividade física moderada estimula o desenvolvimento ósseo, o atraso puberal resultante do treinamento físico vigoroso pode comprometer a aquisição da massa óssea ideal. Em adolescentes do sexo feminino, o excesso de exercício pode causar hipoestrogenismo, com redução do ganho de massa óssea, o que pode ser, em casos graves, irreversível a despeito do retorno da menstruação, da reposição estrogênica e da suplementação com cálcio ${ }^{(4,7,10)}$. A redução da densidade mineral óssea também pode ser observada em adolescentes do sexo masculino submetidos à atividade física extenuante ${ }^{(9)}$. A menor densidade mineral óssea aumenta o risco de fraturas de estresse e de instabilidade da coluna vertebral, com desenvolvimento de escoliose ${ }^{(3)}$.

\section{Atividade física, esportes e crescimento estatural}

Profissionais de saúde são questionados frequentemete quanto aos efeitos positivos do exercício físico sobre o crescimento de seus filhos. Embora muito se especule a esse respeito, existem poucos trabalhos que sustentem tal afirmação ${ }^{(11)}$. Isso se deve principalmente à diversidade de fatores que tornam difíceis os estudos comparativos nesta área como, por exemplo, dificuldade de interpretar o impacto dos esportes na adolescência em virtude dos diferentes estágios puberais; grau de restrição dietética utilizada em alguns treinamentos e grande número de atividades físico-desportivas potenciais e suas variadas formas (intensidade, freqüência) de prática ${ }^{(11)}$.

\section{Influência da intensidade da atividade física}

O exercício moderado promove aumento dos níveis circulantes do GH e IGF-1 por meio do estímulo aferente direto do músculo para a adenohipófise, além do estímulo por catecolaminas, lactato, óxido nítrico e mudanças no balanço ácidobásico ${ }^{(12,13)}$. Tal efeito é, portanto, benéfico para o crescimento linear dos indivíduos pré-púberes. Entretanto, o treinamento vigoroso pode reduzir o ganho estatural, sendo esse efeito resultante mais da intensidade e duração do que propriamente do tipo de exercício praticado ${ }^{(14)}$. Como explicação para esses achados, demonstrou-se que a atividade física intensa causa inibição do eixo GH-IGF-1 ${ }^{(15,16)}$. Theintz et al mostraram redução da altura associada à diminuição do IGF-1 em ginastas de elite submetidas a treinamento físico intensivo (22 horas/ semana) e restrição dietética ${ }^{(16)}$. Caine et al chamam atenção para o fato de que o excesso de atividade física (36 horas/semana) em crianças pré-púberes pode comprometer a estatura final ${ }^{(17)}$. Apesar de crianças geralmente não participarem de esportes competitivos de elite, tais informações devem servir de alerta para os efeitos negativos desse tipo de atividade ${ }^{(17)}$.

Mais recentemente, demonstrou-se o papel das citocinas pró-inflamatórias sobre a atividade hormonal em situações de déficit energético crônico resultante da combinação de atividade física intensa e baixa ingestão calórica ${ }^{(3,18)}$. A prática esportiva competitiva e intensa associada a um gasto energético excessivo estimula a liberação de citocinas, como interleucina-1 (IL-1), IL-6 e fator de necrose tumoral alfa (TNF- $\alpha$ ), os quais poderiam inibir o eixo GH/IGF-1 $1^{(18,19)}$. Guy e Mitchell relataram que a atividade física intensa leva à redução dos níveis séricos do IGF-1, o que poderia vir a comprometer o crescimento e, eventualmente, reduzir a previsão de altura final ${ }^{(15)}$. Esse efeito foi observado até mesmo em treinamentos curtos como uma sessão de uma hora e 50 minutos de pólo aquático ou de luta ${ }^{(13,18)}$. Embora se saiba que o estado catabólico induzido pelo exercício intenso seja o mecanismo desencadeador da liberação destas citocinas, pouco se sabe sobre seus mecanismos fisiopatológicos, com a possível exceção da elevação da IGFBP-I (proteína transportadora do IGF-1), que reduziria a biodisponibilidade do IGF-1 ${ }^{(11)}$. Nemet et al especulam que, à medida que o individuo se adapta à atividade física, as citocinas pró-inflamatórias diminuem, levando a uma reativação do eixo GH-IGF-1 de modo que os níveis de IGF-1 alcancem valores superiores ao pré-treinamento ${ }^{(18)}$.

O Quadro 1 mostra as alterações hormonais que ocorrem em situações de balanço energético negativo. As alterações endócrinas (hipoinsulinemia, hipotriidotironinemia, hipercortisolemia, hipoleptinemia) são indicativas de uma resposta adaptativa ao custo energético (efeito catabólico) do treinamento físico exagerado ${ }^{(3,10,20)}$. 
Quadro 1 - Alterações hormonais em situações de balanço energético negativo (Adaptado de Stafford, $2005^{(3)}$ ).

\begin{tabular}{|lcc|}
\hline Órgão & Hormônio & Nível sérico \\
\hline Tireóide & T3 total & Diminuído \\
Adrenal & Cortisol & Aumentado \\
& Catecolaminas & Aumentado \\
Pâncreas & Insulina & Diminuída \\
& Glucagon & Aumentado \\
Pituitária & Hormônio do crescimento & Aumentado \\
Fígado & IGF-1 & Diminuído \\
& IGFBP-1 & Aumentado \\
Adipócitos e hipotálamo & Leptina & Diminuída \\
Trato gastrointestinal & Grelina & Aumentada \\
\hline
\end{tabular}

IGF-1: fator de crescimento insuliniforme tipo 1; IGFBP-1: proteína transportadora do IGF-1; T3: triiodotironina.

\section{Influência do tipo de atividade física}

Existe uma noção comum entre profissionais de saúde, professores de educação física e pais de que determinados esportes aumentam a estatura, enquanto outros diminuem ${ }^{(21)}$. Entretanto, a literatura não traz evidências que subsidiem tal noção. $\mathrm{O}$ fato de indivíduos altos jogarem basquete e baixos praticarem ginástica se deve tão somente a um viés de seleção no qual os atletas são escolhidos com base no biótipo que possa levá-los a melhores resultados ${ }^{(11,22,23)}$. Ou seja, jogar basquete não acelera o crescimento e praticar ginástica olímpica não retarda o ganho estatural ${ }^{(24)}$. Vários trabalhos com ginastas de ambos os sexos não indicaram efeitos negativos sobre a altura ${ }^{(14,25-27)}$. Damsgaard et al concluíram que, embora nadadores sejam mais altos que ginastas, tal diferença se deve apenas a fatores constitucionais que favorecem e direcionam a escolha do esporte ${ }^{(28)}$. Eisenmann et al avaliaram a estatura final e a taxa de crescimento estimada de corredores durante a infância e adolescência e concluíram que esta atividade não influenciou a previsão de altura final ${ }^{(24)}$. Peltenburg et al, citados por Naughton et al, relatam que alguns atletas de elite já apresentavam baixa estatura dois anos antes de iniciarem sua carreira esportiva ${ }^{(29)}$. Damsgaard et al sintetizam os achados mostrando que o crescimento prépúbere não é adversamente afetado por diferentes esportes e que a seleção da criança para determinado esporte depende de fatores constitucionais ${ }^{(30)}$.

\section{Influência da alimentação}

A restrição dietética utilizada em alguns esportes nos quais a classificação em categorias depende do peso ou nos quais o peso mais baixo se associa à melhor performance (por exemplo, balé, ginástica olímpica, artes marciais) é prejudicial por possibilitar o atraso do crescimento, retardar o desenvolvimento puberal, causar osteopenia, anemia e síndromes relacionadas à deficiência de vitaminas, minerais, aminoácidos, ácidos graxos e elementos traço ${ }^{(11,23)}$.

Enquanto alguns atletas restringem sua alimentação, outros usam aminoácidos como arginina, lisina e ornitina para estimular a secreção de $\mathrm{GH}$, acreditando que tal prática promova um maior ganho de massa e força muscular. No entanto, não existem estudos que comprovem a eficácia dessa prática. A resposta do $\mathrm{GH}$ à administração de aminoácidos apresenta variabilidade individual, sendo alterada pela intensidade da atividade (maior liberação de GH quando o treinamento é mais intenso), sexo (menor resposta em homens), dieta (resposta menor nos que consomem muita proteína) e via de administração (a dose oral necessária para aumentar a liberação de GH geralmente causa desconforto estomacal e diarréia) ${ }^{(31)}$. Além disso, os níveis circulantes de IGF-1 não são alterados após a administração oral de arginina e lisina ${ }^{(31)}$. Portanto, o uso de aminoácidos específicos como estimuladores para a liberação de $\mathrm{GH}$ com o intuito de aumentar o ganho de massa e força muscular ou alterar a composição corporal não é recomendado.

\section{Influência de medicamentos}

- Hormônio de crescimento recombinante humano (rHGH): devido à disponibilidade praticamente universal do rHGH, os indivíduos ansiosos por um maior crescimento estatural, força muscular e aumento de massa magra têm feito uso desse medicamento. Esse desejo vem do conhecimento das ações metabólicas do GH, tais quais: promoção da síntese protéica, antagonismo da ação insulínica, estímulo da lipólise no tecido adiposo, balanço positivo do cálcio, fósforo e magnésio e retenção de sódio, cloro e potássio ${ }^{(12)}$. Apesar disso, o rHGH não é recomendado para melhorar o desempenho atlético. 
Seu uso indiscriminado e abusivo se associa à artralgia, artrite, cardiomegalia, fraqueza muscular, hiperlipidemia, regulação glicêmica prejudicada, risco de diabetes e impotência ${ }^{(12)}$.

- Esteróides anabólicos: observa-se aumento na utilização de esteróides anabólicos por adolescentes ${ }^{(32)}$. A Academia Americana de Pediatria condena seu uso, embora reconheça que aumentem a força muscular. Por isso, muitos usuários justificam seu emprego como otimizador do desempenho atlético. Entretanto, seu emprego está associado a inúmeros efeitos adversos: elevação das enzimas hepáticas, aumento do risco de fenômenos trombóticos (infarto do miocárdio, acidente vascular cerebral), fechamento precoce das cartilagens de crescimento em indivíduos pré-púberes, masculinização nas meninas (hirsutismo, acne, hipertrofia de clitóris) e efeitos indesejáveis nos meninos (calvície precoce, ginecomastia, atrofia testicular, redução da espermatogênese) ${ }^{(32)}$.

\section{Atividade física, esportes e desenvolvimento puberal}

A atividade física vigorosa e extenuante associada à redução da disponibilidade energética pode levar a efeitos adversos sobre o desenvolvimento puberal e a função reprodutiva. Os mecanismos hormonais responsáveis por esses distúrbios são semelhantes aos observados em situações de balanço energético negativo, nas quais ocorre supressão da secreção pulsátil do GnRH, o que causa deficiência na produção dos esteróides sexuais ${ }^{(33)}$. Os principais mecanismos fisiopatológicos envolvidos nessa disfunção são: diminuição da concentração de leptina sérica ${ }^{(3,10,17,34)}$, aumento dos níveis séricos de grelina ${ }^{(3,35)}$, beta-endorfinas ${ }^{(33)}$, fator liberador de corticotrofina (CRF) e de hormônio adrenocorticotrófico $(\mathrm{ACTH})^{(3)}$, além de uma diminuição acentuada da gordura corporal como resultado dos transtornos alimentares. Estima-se que a quantidade de gordura corporal necessária para manter o ciclo menstrual gire em torno de $22 \%$, sendo que $17 \%$ seria a quantidade mínima de gordura para desencadear a menarca ${ }^{(33)}$.

Crianças pré-púberes submetidas à atividade física intensa podem retardar o início da puberdade ${ }^{(26,33)}$. Apesar dessas evidências, em algumas situações é questionável se o atraso puberal é devido à predisposição genética e consequente préseleção ou ao efeito do início precoce de intenso treinamento físico. Atletas oriundas de famílias com predisposição genética para menarca tardia são mais propensas a serem bem- sucedidas em esportes como a ginástica olímpica, já que essa maturação atrasada pode favorecer o desempenho ${ }^{(14,36)}$.

As meninas e adolescentes atletas podem apresentar um continuum de distúrbios menstruais que variam de atraso puberal, menarca tardia, ciclos anovulatórios por deficiência da fase lútea ou insensibilidade hipofisária aos estrógenos a oligomenorréia e amenorréia ${ }^{(3,10,20)}$. Tais distúrbios dependem diretamente da intensidade e duração do exercício, da perda de peso e gordura corporal e do balanço energético negativo ${ }^{(3,33)}$. A amenorréia da mulher atleta é de causa hipotalâmica e depende da intensidade, do tipo da atividade física e da quantidade de gordura corporal ${ }^{(3)}$. Essas anormalidades são mais freqüentes em praticantes de ginástica, balé e corrida de longa distância, nos quais há grande ênfase no baixo peso corpóreo ${ }^{(3)}$. Atualmente, a amenorréia hipotalâmica é considerada uma importante causa de perda óssea prematura quando associada à tríade da mulher atleta: transtorno alimentar, amenorréia e osteoporose ${ }^{(33)}$.

No sexo masculino, os estudos não têm indicado evidências de efeitos adversos na maturação sexual relacionados a treino esportivo. Aparentemente, meninos atletas não têm atraso do desenvolvimento puberal, quando comparados a adolescentes não-atletas ${ }^{(20)}$.

\section{Benefícios e riscos da atividade física e esportes em Pediatria}

Os efeitos benéficos da atividade física são evidenciados nos mais variados órgãos e sistemas: cardiovascular (aumento do consumo de oxigênio, manutenção de boa freqüência cardíaca e volume de ejeção), respiratório (aumento dos parâmetros ventilatórios funcionais), muscular (aumento de massa, força e resistência), esquelético (aumento do conteúdo de cálcio e mineralização óssea), cartilaginoso (aumento da espessura da cartilagem, com maior proteção articular) e endócrino (aumento da sensibilidade insulínica, melhora do perfil lipídico) $)^{(1)}$.

A atividade física também pode prejudicar o crescimento e o desenvolvimento de acordo com sua intensidade, presença de fatores estressantes como competições e lesões, gasto energético, idade e estado nutricional ${ }^{(37,38)}$. Além disso, diferentes esportes se associam a diferentes lesões, não havendo possibilidade de comparação ${ }^{(39)}$.

O principal risco da atividade física ou esporte inadequado são as lesões músculo-esqueléticas: fraturas, osteocondroses, tendinite, escoliose, osteocondrite, espondilose e espondilolistese ${ }^{(20)}$. As cartilagens de crescimento se fecham 
em épocas diversas, estando, portanto, mais vulneráveis a lesões de acordo com a etapa do desenvolvimento (pré-puberal versus puberal) $)^{(1)}$.

As osteocondroses afetam o esqueleto em crescimento e a cartilagem $\operatorname{articular}^{(1)}$. A apofisite de tração é a mais freqüente delas, causada pela combinação do crescimento e do excesso de carga na cartilagem de crescimento devido à tração da unidade músculo-tendão ${ }^{(1,20)}$. As duas apofisites mais comuns são a doença de Osgood-Schlatter (tuberosidade tibial) e a doença de Sever (calcâneo) $)^{(20)}$.

$\mathrm{O}$ pico de fraturas em pediatria coincide com o estirão puberal, talvez decorrente do aumento da remodelação do osso cortical nessa fase do crescimento ${ }^{(1)}$. Por isso, as atividades de carga, embora possam evitar fraturas na vida adulta, em crianças pré-púberes estão mais associadas ao risco de fratura $^{(1)}$.

Os benefícios e riscos associados à participação de crianças em esportes estão descritos no Quadro 2.

\section{Atividade física e esportes para crianças e adolescentes}

Um estudo realizado no Rio Grande do Sul avaliou a participação dos adolescentes em atividades físicas, de acordo com o gênero e a modalidade de esporte ${ }^{(2)}$. Entre os participantes do sexo masculino, as quatro atividades mais realizadas foram: futebol $(82,5 \%)$, voleibol $(18,2 \%)$, musculação $(10,8 \%)$ e atletismo $(10,7 \%)$. Entre as participantes do sexo feminino, as atividades foram: voleibol $(46,3 \%)$, dança $(25,4 \%)$, handebol $(20,3 \%)$ e ginástica $(18,25 \%)$. Os esportes marciais (judô, karatê, boxe, capoeira, jiu-jitsu, taekwondo e kung-fu) eram praticados por $8,5 \%$ dos homens e por apenas $1,9 \%$ das mulheres, enquanto a dança era praticada por $25,4 \%$ das mulheres e apenas 3,8\% dos homens.

Infelizmente, na maioria das vezes o treinamento físico oferecido a crianças é estruturado com base nos modelos utilizados para adultos, isto é, centrados na competição, no êxito e na seletividade ${ }^{(40)}$. Além disso, não se levam em consideração características próprias das diversas faixas etárias, como as proporções corpóreas diferentes (por exemplo, extremidades curtas em relação ao comprimento) e a menor massa muscular, com conseqüente resposta reduzida às atividades que necessitem de força ${ }^{(41)}$. A seleção do esporte mais indicado para cada criança ou adolescente depende de idade, gênero, estágio de desenvolvimento puberal, estado nutricional, presença de limitações físicas ou mentais, maturidade emocional, situação econômica da família, disponibilidade e facilidade de participação na atividade escolhida ${ }^{(42)}$.

Meninos e meninas pré-púberes têm condições físicas comparáveis para a prática de esportes ${ }^{(41)}$. Ambos são semelhantes em termos de altura, peso, porcentagem de gordura corporal, habilidades motoras, força e resistência muscular e níveis séricos de hemoglobina ${ }^{(41)}$. Assim, meninos e meninas podem participar de atividades esportivas juntos até a idade de dez anos. A partir da puberdade, as meninas tendem a ser menores, com ombros mais estreitos, maior porcentagem de gordura corporal (23-27\% para mulheres versus $13-15 \%$ para homens) e apresentam menos força muscular que os adolescentes do sexo masculino, o que requer uma separação de gêneros em esportes competitivos ${ }^{(41)}$. Algumas recomendações para a prática desportiva saudável da criança são mostradas no Quadro $3^{(20,40,42,43)}$. A discussão detalhada sobre as indicações, riscos e benefícios das variadas atividades físicas e esportes fogem ao escopo deste artigo. Entretanto, devido à grande polêmica sobre musculação, esse tema é detalhado a seguir.

Musculação (weight-training) refere-se a contrações musculares repetitivas, com sobrecargas progressivas em um nível submáximo, enquanto halterofilismo (weight-lifting) é definido como um esporte competitivo no qual as contrações musculares são realizadas em carga máxima ${ }^{(1)}$. Entretanto, na prática, muitas crianças e adolescentes realizam musculação sem orientação adequada, com carga máxima e por tempo prolongado, à semelhança do halterofilismo.

Quadro 2 - Benefícios e riscos físicos associados à participação de crianças em esportes.

\begin{tabular}{|ll|}
\hline Benefícios & Riscos \\
\hline Promoção de crescimento físico & Lesões musculares \\
Estímulo do desenvolvimento motor & Trauma \\
Benefício cardiovascular & Osteocondrose (apofisite de tração) \\
Efeito positivo no perfil lipídico & Fratura \\
Redução do risco de diabetes melito tipo 2 & Disfunção menstrual \\
Incremento da massa óssea & Escoliose \\
Aumento de força e massa muscular & Tendinite \\
\hline
\end{tabular}


Quadro 3 - Recomendações para a prática esportiva saudável de crianças e adolescentes.

Encorajar crianças a participar de esportes consistentes com suas habilidades e interesses

Orientar atividade física apropriada para crianças e adolescentes com necessidades especiais ou limitações

Acompanhar crescimento (peso, altura) e evolução do desenvolvimento puberal

Assegurar dieta balanceada com aporte calórico, de cálcio e de ferro adequados

Prover condições de exercício seguras

Ficar atento para complicações físicas (tendinites, apofisites) e emocionais (transtornos alimentares, distúrbios do sono, ansiedade)

Aumentar o tempo de recuperação quando realizar atividades físicas de carga elevada

Priorizar as atividades aeróbicas em lugar das anaeróbicas

Evitar situações nas quais sejam necessárias apnéias prolongadas (mergulho, halterofilismo)

No treinamento de força, evitar cargas elevadas sobre a coluna

Nas atividades que exijam alta coordenação motora, ter em mente o desenvolvimento psicomotor da criança

Priorizar movimentos e habilidades naturais no lugar de exercícios elaborados

A prática de musculação por crianças e no início da adolescência é um tópico controverso. Alguns autores afirmam ser essa atividade prejudicial a pré-adolescentes e outros mostram que pode ser benéfica se bem supervisionada ${ }^{(44-}$ 46). Aqueles que contra-indicam a prática por jovens prépúberes argumentam que, além de não aumentar a força muscular devido à quantidade insuficiente de andrógenos circulantes, ela ainda se associa a um potencial risco de lesão da cartilagem de crescimento e de fechamento precoce das epífises, como resultado da sobrecarga excessivaa ${ }^{(47)}$. Isto é particularmente importante em crianças com baixa estatura que, na tentativa de compensar seu déficit estatural com o aumento da massa muscular, podem prejudicar ainda mais seu potencial de crescimento. $\mathrm{O}$ efeito benéfico e seguro da musculação em crianças é relatado apenas em programas experimentais de treinamento que utilizam pesos e aparelhos isotônicos sob supervisão de instrutores, com frequiência de duas a três vezes por semana, durante seis semanas a 21 meses $^{(47)}$. Nesses casos, mesmo em crianças pré-púberes, ocorre um aumento de força e resistência muscular em resposta a adaptações neuromusculares, na ausência de hipertrofia muscular, com baixo risco de lesão e ausência de impacto negativo sobre o crescimento ${ }^{(1,45,47,48)}$. Uma vez suspenso o treinamento, observa-se perda de toda a força muscular adquirida durante os treinos ${ }^{(20,48)}$. Outros estudos mostram que exercícios de alta repetição (13 a 15 repetições) e moderada resistência produzem maior ganho de força do que os de baixa freqüência (seis a oito repetições) e alta intensidade ${ }^{(45)}$.

Em adolescentes pós-púberes ou no final da adolescência, os exercícios isométricos de resistência se associam a benefícios para o conteúdo mineral ósseo, a composição corporal e a redução do risco de lesões relacionadas ao esporte ${ }^{(47)}$.
A Academia Americana de Pediatria admite a realização de treinamentos de força e resistência para crianças, desde que programados de forma adequada em termos de freqüência, tipo, intensidade e duração ${ }^{(48)}$. Cargas máximas só devem ser realizadas por adolescentes que tenham atingido estágio puberal 5 de Tanner ${ }^{(48)}$.

\section{Discussão}

Existe grande dificuldade em avaliar e interpretar os estudos do impacto da atividade física e esportes sobre o crescimento e desenvolvimento de crianças e adolescentes devido a diversos fatores. Intervêm na avaliação desse impacto fatores relacionados à faixa etária, tais quais as diferentes fases de crescimento (repleção, estirão) e os diversos estágios puberais, fatores relacionados à diversidade de atividades físico-desportivas realizadas como vôlei, natação e ciclismo, bem como a forma de praticá-las (competitivamente ou não), fatores relacionados à adoção de restrição dietética, ao uso de suplementos alimentares e à utilização de substâncias ergogênicas e esteródes anabólicos, além da diversidade metodológica dos trabalhos sobre o tema ${ }^{(11)}$. Nesta revisão, o impacto da atividade física e esportes foi avaliado em relação a três questões: mineralização óssea, crescimento estatural e desenvolvimento puberal.

Os resultados mostram que, embora a atividade física e os esportes sejam benéficos para o aumento da densidade mineral óssea, principalmente o treinamento de força com impacto realizado próximo à puberdade, o seu excesso pode ter um efeito oposto, promovendo osteoporose e aumentando o risco de fraturas ${ }^{(1,4,9)}$. Dentre os fatores endógenos envolvidos no processo da osteogênese induzido pelos exercícios, 
ressalta-se a necessidade de mais estudos sobre a importância da leptina e dos marcadores de remodelação óssea ${ }^{(6)}$.

$\mathrm{O}$ crescimento linear ou estatural é potencializado pela prática de atividade física moderada, a qual, entre outros mecanismos, aumenta os níveis séricos dos principais hormônios promotores do crescimento ${ }^{(12,13)}$. Similarmente ao que ocorre com a densidade mineral óssea, a atividade física extenuante reduz os níveis desses hormônios, podendo inclusive comprometer a altura final ${ }^{(14-19)}$. Não parece haver dúvidas de que as diferentes estaturas observadas em participantes de determinados esportes deve-se a apenas um viés de seleção e não ao tipo de esporte praticado ${ }^{(11,22-24,28)}$. Tanto a restrição dietética quanto o uso indiscriminado de suplementos alimentares ou substâncias ergogênicas são prejudiciais para crianças e adolescentes envolvidos em atividades físicodesportivas $^{(11,23,31,32)}$.

Em relação ao impacto dos esportes sobre o desenvolvimento puberal, os trabalhos revisados mostram que, quando realizados de forma vigorosa, podem levar ao atraso puberal e a distúrbios reprodutivos ${ }^{(33-35)}$. Apesar disso, alguns autores questionam se o atraso puberal deve-ser-ia, na realidade, à predisposição familiar ${ }^{(14,36)}$. As meninas são as principais afetadas pelos distúrbios puberais e reprodutivos relaciona-

\section{Referências bibliográficas}

1. Broderick CR, Winter GJ, Allan RM. Sport for special groups. Med J Aust 2006;184:297-302.

2. Azevedo MR, Araújo CL, Cozzensa da Silva MC, Hallal PC. Tracking of physical activity from adolescence to adulthood: a population-based study. Rev Saude Publica 2007;41:69-75.

3. Stafford DE. Altered hypothalamic-pituitary-ovarian axis function in young female athletes. Treat Endocrinol. 2005;4:147-54.

4. Eliakim A, Beyth Y. Exercise training, menstrual irregularities and bone development in children and adolescents. J Pediatr Adolesc Gynecol 2003;16:201-6.

5. Lima F, De Falco V, Baima J, Carazzato JG, Pereira RM. Effect of impact load and active load on bone metabolism and body composition of adolescent athletes. Med Sci Sports Exerc 2001;33:1318-23.

6. Borba VZ, Kulak CA, Lazaretti-Castro M. Neuroendocrine control of bone mass: myth or reality? Arq Bras Endocrinol Metab 2003;47:453-7.

7. Muñoz MT, de la Piedra C, Barrios V, Garrido G, Argente J. Changes in bone density and bone markers in rhythmic gymnasts and ballet dancers: implications for puberty and leptin levels. Eur J Endocrinol 2004;151:491-6.

8. Pettersson U, Nordström P, Alfredson $\mathrm{H}$, Henriksson-Larsén $\mathrm{K}$, Lorentzon R. Effect of high impact activity on bone mass and size in adolescent female: a comparative study between two different types of sports. Calcif Tissue Int 2000;67:207-14

9. Silva CC, Teixeira AS, Goldberg TB. O esporte e suas implicações na saúde óssea de atletas adolescentes. Rev Bras Med Esporte 2003;9:426-32.

10. No authors listed. American Academy of Pediatrics. Committee on Sports Medicine and Fitness. Medical concerns in the female athlete. Pediatrics 2000;106:610-3. dos à prática inadequada da atividade física ${ }^{(3,33)}$. Não foram encontrados estudos indicando tais efeitos em meninos ${ }^{(20)}$.

\section{Considerações finais}

A atividade física leve a moderada tem efeito benéfico sobre o crescimento estatural e o desenvolvimento ósseo, enquanto a atividade física intensa atenua o crescimento, podendo causar atraso puberal e diminuição da mineralização esquelética. Diferentes modalidades esportivas não têm efeitos específicos no sentido de aumentar ou diminuir a altura final. O que ocorre é um viés de seleção no qual crianças com baixa estatura são recrutadas para alguns esportes (ginástica olímpica) e as de alta estatura para outros (basquete) em função do um possível melhor desempenho na atividade. Embora seja um tema controverso, a prática de musculação por crianças pré-púberes não é recomendada, a não ser sob supervisão especializada. Como não é possível saber se pais, técnicos e professores podem supervisioná-los, a musculação, embora permitida, deve ser recomendada com cautela para pré-adolescentes. As demais atividades físicas aeróbicas serão indicadas a depender da idade, sexo, estágio puberal e estado de saúde da criança.

11. Silva CC, Goldberg TB, Teixeira AS, Marques I. Does physical exercise increase or compromise children's and adolescent's linear growth? Is it a myth or truth? Rev Bras Med Esporte 2004;10:520-4.

12. Godfrey RJ, Madgwick Z, Whyte GP. The exercise-induced growth hormone response in athletes. Sports Med 2003;33:599-613.

13. Nemet D, Rose-Gottron CM, Mills PJ, Cooper DM. Effect of water polo practice on cytokines, growth mediators and leukocytes in girls. Med Sci Sports Exerc 2003;35:356-63.

14. Georgopoulos NA, Markou K, Theodoropoulou A, Paraskevopoulou P, Varaki L, Kazantzi Z et al. Growth and pubertal development in elite female rhythmic gymnasts. J Clin Endocrinol Metab 1999;84:4525-30.

15. Guy JA, Micheli LJ. Strength training for children and adolescents. J Am Acad Orthop Surg 2001;9:29-36.

16. Theintz GE, Howald H, Weiss $U$, Sizonenko PC. Evidence for a reduction of growth potential in adolescents female gymnasts. J Pediatr 1993;122:306-13.

17. Caine D, Lewis R, O'Connor P, Howe W, Bass S. Does gymnastics training inhibit growth of females? Clin J Sport Med 2001;11:260-70.

18. Nemet D, Oh Y, Kim HS, Hill M, Cooper DM. Effect of intense exercise on inflammatory cytokines and growth mediators in adolescent boys. Pediatrics 2002;110:681-9.

19. Scheett TP, Nemet D, Stoppanni J, Maresh CM, Newcomb R, Cooper DM. The effect of endurance-type exrecice training on growth mediators an inflammatory cytokines in pre-pubertal and early pubertal males. Pediatr Res 2002;52:491-7.

20. No authors listed. Intensive training and sports specialization in young athletes. American Academy of Pediatrics. Committee on Sports Medicine and Fitness. Intensive training and sports specialization in young athletes. Pediatrics 2000;106:154-7. 
21. Metzl JD. Expectations of pediatric sport participation among pediatricians, patients, and parents. Pediatr Clin North Am 2002;49:497-504.

22. Bass S, Bradney M, Pearce G, Hendrich E, Inge K, Stuckey S et al. Short stature and delayed puberty in gymnasts: influence of selection bias on leg length and the duration of training on trunk length. J Pediatr 2000;136: 149-55.

23. Rogol AD, Clark PA, Roemmich JN. Growth and pubertal development in children and adolescents: effects of diet and physical activity. Am J Clin Nutr 2000;72(2 Suppl):521S-8S

24. Eisenmann JC, Malina RM. Growth status and estimated growth rate of young distance runners. Int J Sports Med 2002;23:168-73.

25. Daly RM, Rich PA, Klein R, Bass SL. Short stature in competitive prepubertal and early pubertal male gymnasts: the result of selection bias or intense training? J Pediatr. 2000;137:510-6.

26. Gurd B, Klentrou P. Physical and pubertal development in young male gymnasts. J Appl Physiol 2003;95:1011-5.

27. Bass S, Bradney M, Pearce G, Hendrich E, Inge K, Stuckey S et al. Short stature and delayed puberty in gymnasts: influence of selection bias on leg length and the duration of training on trunk length. J Pediatr 2000;136:149-55.

28. Damsgaard R, Bencke J, Matthiesen G, Petersen JH, Müller J. Body proportions, body composition and pubertal development of children in competitive sports. Scand J Med Sci Sports 2001;11:54-60.

29. Naughton G, Farpour-Lambert NJ, Carlson J, Bradney M, Van Praagh E. Physiological issues surrounding the performance of adolescent athletes. Sports Med 2000;30:309-25.

30. Damsgaard R, Bencke J, Matthiesen G, Petersen JH, Müller J. Is prepubertal growth adversely affected by sport? Med Sci Sports Exerc 2000;32: 1698-1703.

31. Chromiak JA, Antonio J. Use of amino acids as growth hormone-releasing agents by athletes. Nutrition. 2002;18:657-61.

32. No authors listed. Adolescents and anabolic steroids: a subject review. American Academy of Pediatrics. Committee on Sports Medicine and Fitness. Pediatrics 1997:99:904-8.

33. Pardini DP. Alterações hormonais da mulher atleta. Arq Bras Endocrinol Metab 2001;45:343-51.

34. Welt CK, Chan JL, Bullen J, Murphy R, Smith P, DePaoli AM et al. Recombinant human leptin in women with hypothalamic amenorrhea. N Engl J Med 2004;351:987-97.
35. De Souza MJ, Leidy HJ, O'Donnell E, Lasley B, Williams NI. Fasting ghrelin levels in physically active women: relatiosnhip with menstrual disturbances and metabolic hormones. J Clin Endocrinol Metab 2004;89:3536-42.

36. Georgopoulos NA, Markou KB, Theodoropoulou A, Vagenakis GA, Benardot D, Leglise $\mathrm{M}$ et al. Height velocity and skeletal maturation in elite female rhythmic gymnasts. J Clin Endocrinol Metab 2001;86:5159-64.

37. Maron BJ, Chaitman BR, Ackerman MJ, Bayés de Luna A, Corrado D, Crosson JE et al; Working Groups of the American Heart Association Committee on Exercise, Cardiac Rehabilitation, and Prevention;Councils on Clinical Cardiology and Cardiovascular Disease in the Young. Recommendations for physical activity and recreational sports participation for young patients with genetic cardiovascular diseases. Circulation 2004;109:2807-16.

38. Stricker PR. Sports training issues for the pediatric athlete. Pediatr Clin North Am 2002;49:793-802.

39. DeHaven KE, Lintner DM. Athletic injuries: comparison by age, sport, and gender. Am J Sports Med 1986;14:218-24.

40. Futsal Brasil [homepage on the Internet]. A criança submetida precocemente ao esporte: benefícios e malefícios [Cited 2007 Jun 9]. Available from: http:// www.futsalbrasil.com.br/artigos/artigo.php?cd_artigo153.

41. Greydanus DE, Patel DR. The female athlete. Before and beyond puberty. Pediatr Clin North Am 2002;49:553-80.

42. Sociedade Brasileira de Cardiologia. Diretriz de prevenção da aterosclerose na infância e adolescência. Arq Bras Cardiol 2005;85 (Suppl 6):1-36.

43. No authors listed. Physical fitness and activity in schools. American Academy of Pediatrics. Pediatrics 2000;105:1156-7.

44. Rians CB, Weltman A, Cahill BR, Janney CA, Tippett SR, Katch FI. Strenght training for prepubescent males: is it safe? Am J Sports Med 1987;15:483-9.

45. Faigenbaum AD, Westcott WL, Loud RL, Long C. The effects of different resistance training protocols on muscular strength and endurance development in children. Pediatrics 1999; 104:e5.

46. Faigenbaum $A D$. Strength training for children and adolescents. Clin Sports Med 2000;19:593-619.

47. Malina RM. Weight training in youth-growth, maturation, and safety: an evidence-based review. Clin J Sport Med 2006;16:478-87.

48. American Academy of Pediatrics Council on Sports Medicine and Fitness, McCambridge TM, Stricker PR. Strength training by children and adolescents. Pediatrics 2008;121:835-40 